\title{
A Comparison of Two Immunoassays for Analysing Plasma 25- hydroxyvitamin D
}

\author{
Kurtis Sarafin, Nicolas Hidiroglou and Stephen P.J. Brooks*
}

\author{
Nutrition Research Division, Health Canada, Ottawa, Canada
}

\begin{abstract}
A total of 1628 human plasma samples from Cycle 1 of the Canadian Health Measures Survey were assayed for total 25-hydroxyvitamin D using the DiaSorin RIA method and the Diasorin "LIAISON 25 OH Vitamin D Total" method. Bland-Altman comparison showed an average bias of $4.8 \pm 16.7 \mathrm{nmol} / \mathrm{L}(6.3 \%$ : $\mathrm{P}<0.001)$ with the LIAISON method giving higher values. The relationship was investigated using linear and Deming regression. Linear regression gave: LIAISON $=\operatorname{RIA}^{*}(0.87 \pm 0.02)+(13.3 \pm 1.2)($ mean $\pm \mathrm{SE})$ and weighted Deming regression (constant CV) gave: LIAISON $=$ RIA $*(1.14 \pm 0.02)-(4.2 \pm 1.2)$. The significant deviations from a slope of unity and the significant non-zero intercepts were further investigated using non-linear regression. Quadratic regression gave: LIAISON $=\mathrm{RIA}^{2} *(-0.0025 \pm$ $0.0005)+\operatorname{RIA} *(1.211 \pm 0.07)+(2.9 \pm 2.5)$. The intercept was not significantly different from 0 . The quadratic equation significantly decreased the residual sum of squares $(\mathrm{P}<0.0001)$ indicating this model better described the relationship. Non-linearity was apparent at RIA 25 -hydroxyvitamin $\mathrm{D} \geq 110 \mathrm{nmol} / \mathrm{L}$, where the relationship was described by: LIAISON $=97.5339+0.1388 *$ RIA $\left(r^{2}=0.0039\right.$; N.S. $)$. However, removing points RIA $\geq 110 \mathrm{nmol} / \mathrm{L}$ did not substantially alter the regression parameters. Comparing the analytical imprecision with the total random regression error $\left(S_{\mathrm{y} / \mathrm{x}}\right)$ suggested that sample-related effects were not present. It is recommended that cross-over analysis between these two methods include points from all parts of the range of interest to elucidate the complete nature of the relationship.
\end{abstract}

Keywords: Vitamin D, Liaison, RIA, regression, comparison.

\section{INTRODUCTION}

Vitamin D is metabolized by the body into several compounds that play a central role in calcium and phosphorous absorption, helping to maintain serum calcium and phosphorus levels through its influence on the small intestine's efficiency to absorb these minerals [1]. Vitamin D intake has been linked to a reduced risk of breast cancer [2], colorectal cancer and adenoma [3], cardiovascular disease [4, 5] and even multiple sclerosis and type 1 diabetes [6]. Many effects are mediated through its ability to act directly on DNA transcription when bound to its receptor [7].

Vitamin D occurs primarily in two forms: cholecalciferol (D3), the form synthesized from exposure to sunlight and found in fortified milk and margarine products in Canada; and ergocalciferol (D2), which is obtained exclusively through the diet and occurs mostly in fungi exposed to UV irradiation [8]. Vitamin D status is normally assessed by measuring the circulating 25-hydroxylated forms of D2 and D3, although the 1,25 dihydroxylated metabolite calcitriol is the predominant active form [9]. Status is important to health professionals because of the link to long-term bone health and potentially to other diseases. For this reason, it is measured routinely during population surveys such as the Canadian Health Measures Survey (CHMS), the National

*Address correspondence to this author at the Nutrition Research Division, E315-PL2203E Banting Research Centre, 251 Sir Frederick Banting Driveway, Ottawa, ON K1A 0K9, Canada; Tel: 613-957-0932; Fax: 613-9410451; E-mail: Steve.Brooks@hc-sc.gc.ca
Health and Nutrition Examination Survey (NHANES) in the United States [10], and several others [11-15].

Difficulties arise, however, when comparing among survey results because of differences between methodologies used to measure 25-hydroxy D2, 25-hydroxy D3, the sum of these two forms $(25(\mathrm{OH}) \mathrm{D})$, as well as other circulating forms. These methodologies include gas or liquid chromatography (usually coupled with a mass spectrometer detector), antibody based immunoassays and the now almost obsolete protein binding assays [16]. Selection of an assay method for a survey is often based on a combination of sample throughput, analytical requirements (only $25(\mathrm{OH}) \mathrm{D}$ or minor metabolites as well), and budget. Both positive and negative performance characteristics are associated with each method. Indirect or direct (extraction or no extraction) antibody based methods are limited by the variability in antibody preparations, the sensitivity of the antibody-antigen binding assay conditions [17], and the nature of the assay method: they do not give a direct measure of $25(\mathrm{OH}) \mathrm{D}$. Advantages of direct antibody methods over indirect include the fact that they are easily applicable to automation and high throughput, they measure total $25(\mathrm{OH}) \mathrm{D}$, and are sensitive enough to be used to monitor the full range of human $25(\mathrm{OH}) \mathrm{D}$ plasma concentrations. Chromatographic techniques have the advantage of being able to identify and quantify multiple vitamin D metabolites and they tend to have less inter- and intra-assay variability but they do not typically allow high sample throughput and often are associated with a high initial overhead cost. 
Canada and the United States have embarked on a joint process to set dietary recommendations common to both countries [18]. As part of this process, it is important to compare the vitamin D status of both countries, a process made difficult because the two national surveys use different methods. As part of the first cross-sectional sampling of the Canadian population, the CHMS offered a unique opportunity to compare a large number of samples, monitored over several months using the Diasorin Liaison method, an automated direct cheminluminescence immunoassay, against a manual indirect radioimmunoassay method, which has been used by the United States national survey (NHANES). Other comparisons between these two methods have included more than one method $[19,20]$ or have reported a significant inconsistency and variability among laboratories [21]. We were interested in assessing performance over several assay kit lots using a large sample size to resolve some of these issues.

\section{MATERIALS AND METHODS}

\section{Manual RIA Method}

Manual radioimmunoassay (RIA) kits for the analysis of $25 \mathrm{OH}$ Vitamin D were obtained from Diasorin Inc, Stillwater, MN, USA. All reagents required to perform the analysis are contained within the RIA kit including; $25(\mathrm{OH}) \mathrm{D}$ calibrators, non-specific binding/addition buffer, 25(OH)D antiserum, 25(OH)D tracer, donkey anti-goat (DAG) precipitating complex, 25(OH)D controls and acetonitrile. The RIA assay was performed as directed by the manufacturer's product insert.

The indirect assay is a two-step procedure: the first step is a liquid-liquid extraction using acetonitrile and $50 \mu \mathrm{L}$ of plasma, and the second step is the immunoassay itself, which consists of an equilibrium competition for the antibody between iodine $^{125}$ labelled 25(OH)D and endogenous 25(OH)D present in the human plasma sample. The primary antibody is specific for both $25(\mathrm{OH}) \mathrm{D}_{2}$ and $25(\mathrm{OH}) \mathrm{D}_{3}$.

The antibody, tracer and $25 \mu \mathrm{L}$ of the sample extract are incubated in $12 \times 75 \mathrm{~mm}$ disposable culture tubes (Fisher Scientific Co., Ottawa, ON, Canada) for 90 minutes at room temperature. A secondary antibody (DAG precipitating complex) was then added and incubated for another 20 minutes to precipitate out the bound material. All excess liquid was decanted and the remaining precipitate was measured using a Wallac 1470 Gamma Counter (Perkin Elmer, Waltham, Massachusetts, USA). The amount of gamma radiation measured is inversely proportional to the concentration of $25(\mathrm{OH}) \mathrm{D}$ present in a given sample. A six point calibration curve, consisting of serum containing known $25(\mathrm{OH}) \mathrm{D}$ concentrations was used to determine the $25(\mathrm{OH}) \mathrm{D}$ content of the unknown samples.

\section{Automated CLIA Method}

This assay is a direct competitive chemiluminescence immunoassay (CLIA), which uses $25(\mathrm{OH}) \mathrm{D}$ conjugated to an isoluminol derivative to compete with the $25(\mathrm{OH}) \mathrm{D}$ present in the plasma. There is no indication of the chemical form of the conjugated $25(\mathrm{OH}) \mathrm{D}$. A LIAISON ${ }^{\circledR}$ autoimmunoanalyzer and LIAISON ${ }^{\circledR} 25 \mathrm{OH}$ Vitamin D TOTAL Assay integrals were obtained from Diasorin Inc, Stillwater, MN,
USA. The integral contains the following reagents required to perform the CLIA: magnetic particles coated with antibody against $25(\mathrm{OH}) \mathrm{D}$, assay buffer, conjugated $25(\mathrm{OH}) \mathrm{D}$ and $25(\mathrm{OH}) \mathrm{D}$ calibrators. Additionally purchased from Diasorin Inc, (Stillwater, MN, USA) to perform the assay were: Liaison system liquid, starter reagents and reaction modules.

The assay was performed as indicated in the manufacturer's product insert. During the first incubation, $25(\mathrm{OH}) \mathrm{D}$ is dissociated from its binding protein and binds to the antibody on the magnetic particles. The antibody is specific for both $25(\mathrm{OH}) \mathrm{D}_{2}$ and $25(\mathrm{OH}) \mathrm{D}_{3}$. After a 10 minute incubation, the conjugated $25(\mathrm{OH}) \mathrm{D}$ is added and allowed to incubate for 10 minutes again. The magnetic particles are then washed of all excess liquid and unbound material. A starter reagent is added to initiate a flash chemiluminescent reaction of the conjugated isoluminol derivative, which is then measured by a photomultiplier. The amount of light measured is inversely proportional to the concentration of $25(\mathrm{OH}) \mathrm{D}$ present in a given sample. A two point calibration curve, consisting of serum containing known $25(\mathrm{OH}) \mathrm{D}$ concentrations, is constructed. Unknowns are obtained by comparison with the calibration curve.

\section{Samples and Method Comparison}

Plasma samples were obtained from the CHMS, a repeated cross sectional survey of the Canadian Population [22]. The first cycle of this survey consisted of 5,604 subjects (48\% males), aged 6-79 y (median age 33 y) who reported to a mobile examination centre for physical measurements. The survey excluded residents of Indian reserves, Crown lands, certain remote regions, institutions, and fulltime members of the Canadian Forces. Data was collected over a period of two years at 15 sites across Canada [23]. The initial survey contained plasma $25(\mathrm{OH}) \mathrm{D}$ data on 5,298 $(94 \%)$ of the subjects (median vitamin $\mathrm{D}$ in males $=66.1$ $\mathrm{nmol} / \mathrm{L}$, females $=67.9 \mathrm{nmol} / \mathrm{L})$.

The present cross-over study used a portion $(31 \%)$ of these samples (1628 samples: Table 1). Of the 3670 excluded samples, 3080 were dropped because they did not have data for both RIA and LIAISON (only sites 1-7 were assayed by both methods). A further 581 were excluded because of problems with external control validation during the RIA measurement, five were excluded because their values fell below the lower range of the RIA $(<6 \mathrm{nmol} / \mathrm{L})$ or the LIAISON measurement $(<10 \mathrm{nmol} / \mathrm{L})$, and four values were greater than 4 standard deviations from the analysed mean of all samples. The final sample contained $48 \%$ males with a median age of 35 for males and 37 for females (range 6-79 y).

The samples were collected in lavender top blood collection tubes containing ethylenediaminetetraacetic acid (EDTA). The plasma was removed on site and shipped to our laboratory frozen on dry ice and then subsequently stored at $-80^{\circ} \mathrm{C}$ until analysis. The samples were analysed by RIA within 3 months of receipt and then stored at $-80^{\circ} \mathrm{C}$ until re-analysis by LIAISON. Samples were analyzed by RIA from March 2007-April 2008 and by LIAISON from February 2009-April 2009. In some cases samples were thawed and refrozen more than once for the RIA assay. These occurred when the RIA quality control parameters indicated 
Table 1. Regression Analysis of RIA vs. LIAISON Measures

\begin{tabular}{|c|c|c|}
\hline Comparison method & Parameter & Value $^{\mathrm{a}}$ \\
\hline \hline Bland-Altman bias: & $\mathrm{nmol} / \mathrm{L}$ & $4.8 \pm 16.7$ \\
& $\%$ & $-3 \pm 25.8$ \\
\hline Weighted Deming Regression & intercept & $1.14 \pm 0.02(\mathrm{p}<0.0001)$ \\
\hline Nonlinear quadratic regression: & slope & $-0.0025 \pm 0.0005(\mathrm{p}<0.0001)$ \\
LIAISON $=\mathrm{a} * \mathrm{RIA}^{2}+\mathrm{b} *$ RIA $+\mathrm{c}$ & $\mathrm{a}$ & $1.21 \pm 0.07(\mathrm{p}<0.0001)$ \\
& $\mathrm{b}$ & $2.9 \pm 2.5(\mathrm{p}=0.2441)$ \\
\hline
\end{tabular}

${ }^{\mathrm{a}}$ value \pm standard error.

that the assay results were too variable or were outside tolerable ranges for the assay.

\section{Quality Control}

Quality controls were included with each analytical run for both the RIA and LIAISON methods. 5 controls were run in total, two levels of controls purchased from Diasorin Inc. (Stillwater, MN, USA) and the other three levels were purchased from Biorad Diagnostics Inc. (Montreal, Quebec, Canada). The laboratory has participated in the Vitamin D External Quality Assessment Scheme (DEQAS) since January 2006 for the RIA method and since January 2008 for the Liaison method and has been in proficient standing for both since enrolment.

\section{Statistical Analysis}

Statistical analysis was performed using Analyse-it for Microsoft Excel (Bland-Altman plots, regression analysis) and Statistica 7.0 (Statsoft, Tulsa, OK, USA). Regression analysis was carried out to evaluate the performance of the methods and to assess whether a linear relationship between the two variables existed. Linear regression was performed to assess whether the observed spread of points across the regression $\left(S_{\mathrm{y} / \mathrm{x}}\right)$ was a function only of the total analytical imprecision $\left(s_{\mathrm{a} \text {,tot }}=\sqrt{S_{\text {RIA }}^{2}+S_{\text {LIAISON }}^{2}}\right.$ ) or whether it included sample-related effects [24]. Total analytical imprecision was assessed using all data from high and low QC material which was purchased from Biorad Diagnostics Inc. These QC materials were analyzed with every run (both methods) during the method comparison. Imprecision for each method was estimated as the average variance across the measured range (37 - $129 \mathrm{nmol} / \mathrm{L}$ for RIA; $29.5-84 \mathrm{nmol} / \mathrm{L}$ for LIAISON) calculated as the square root of the sum of measured variances at the two values. This was necessary because the variance was proportional to the magnitude of the measured value (CVs were constant).

Method comparison was performed using weighted Deming regression (constant $\mathrm{CV}$ ) to account for the nonuniformity of variance across the response range [25]. The assumption of a constant variance ratio was verified using repeated measurements of the QC material described above $(\mathrm{CV}=10.5 \%$ at $37 \mathrm{nmol} / \mathrm{L}$ and $10.8 \%$ at $129 \mathrm{nmol} / \mathrm{L}$ for RIA;
$\mathrm{CV}=11.5 \%$ at $29.5 \mathrm{nmol} / \mathrm{L}$ and $9.8 \%$ at $84 \mathrm{nmol} / \mathrm{L}$ for LIAISON).

The Shapiro-Wilk W test showed that both the LIAISON and RIA data were not normally distributed $(\mathrm{P}<0.0001)$. Both data sets had significantly more observations in the lower portion of the graph than normal (skewness $=0.42$ for RIA and 0.43 for LIAISON). In addition, the LIAISON data had more observations near the media than the RIA data (kurtosis $=0.09$ for RIA and 0.49 for LIAISON). Thus, correlation analysis was performed using the Spearman correlation coefficient, which gave a value of 0.77 (CI: 0.75 to 0.79 ). The data were also fitted by quadratic regression to test the linearity of the response. Agreement between the two methodologies was assessed using a plot of the difference as a function of the mean $25(\mathrm{OH}) \mathrm{D}$ values as described by Bland and Altman [26].

\section{RESULTS}

\section{Samples}

None of the samples showed visible signs of hemolysis or lipemia. Plasma samples were frozen and thawed a maximum of 3 times. Under this treatment, 25(OH)D is stable [16].

\section{Estimates of Precision}

The RIA and Liaison method intra- and inter-assay \%CV was estimated by analysing 5 different samples in duplicate over 3 days. The Liaison method intra and inter assay \% CV ranged from $3.2 \%$ to $8.01 \%$ and $6.9 \%$ to $12.7 \%$, respectively. The RIA method intra and inter assay \% CV ranged from $1.0 \%$ to $13.7 \%$ and $8.1 \%$ to $12.0 \%$, respectively.

\section{Method Comparison}

The RIA method average was $64.0 \pm 23.8$ (median 62.8; $95 \%$ CI from 62.7 to 65.1 ; range $6.7-145.0$ ). The LIAISON method average was $68.8 \pm 26.5$ (median $68.8 ; 95 \%$ CI from 67.6 to 70.1 ; range 10.0-213.4). Differences between the RIA and LIAISON methods were assessed graphically using a Bland-Altman percentage difference vs. average plot (Fig. 1). The overall bias was $4.8 \mathrm{nmol} / \mathrm{L}$ with the LIAISON giving higher values (Table 1). The data was further analysed using weighted Deming regression to determine the linearity of response. This gave a negative intercept and a slope significantly different from unity. Visual inspection of the data 
suggested a non-linear relationship with a plateau at RIA 25(OH)D concentrations in excess of $110 \mathrm{nmol} / \mathrm{L}$ (Fig. 2A). Regression of the 65 samples with concentrations $\geq 110$ $\mathrm{nmol} / \mathrm{L}$ (approximately $4 \%$ of the total data set) gave a nonsignificant slope of $0.14 \pm 0.28\left(r^{2}=0.004\right.$; Fig. 2B). However, removing these points from the regression analysis did not substantially alter the weighted Deming parameters. Linearity was further tested using the Linearity Test (Analyse-It). This revealed that a quadratic equation slightly but significantly improved the fit (Table 1) as shown by a reduction in the sum of squares due to lack of fit (395620 for linear regression vs. 389482 for quadratic regression; $\mathrm{p}<0.0001)$.

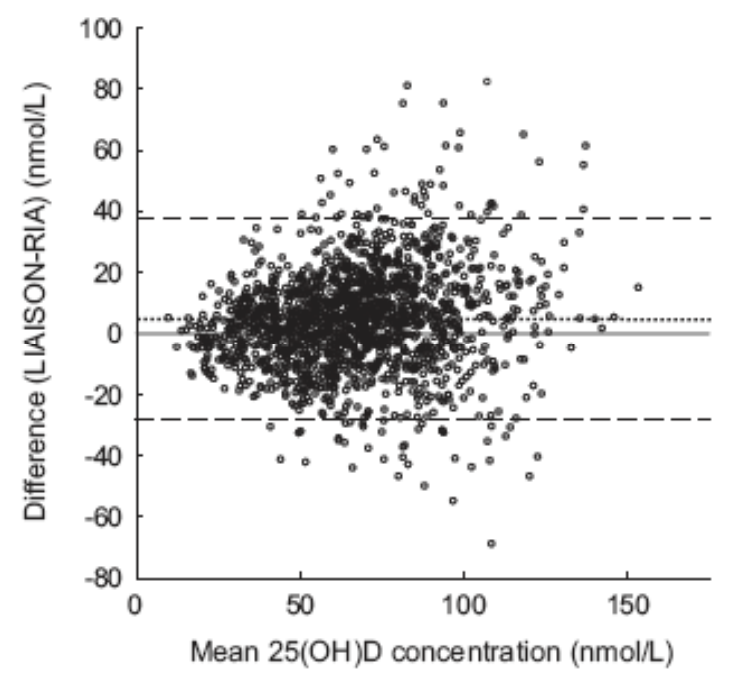

Fig. (1). Bland-Altman plot showing differences between methodologies plotted as a function of the average $25(\mathrm{OH}) \mathrm{D}$ value from both methodologies. Solid line shows identity. Dotted line shows average bias of $4.8 \mathrm{nmol} / \mathrm{L}$. Dashed lines show $95 \%$ limits of agreement (-28.1 to $37.6 \mathrm{nmol} / \mathrm{L})$.

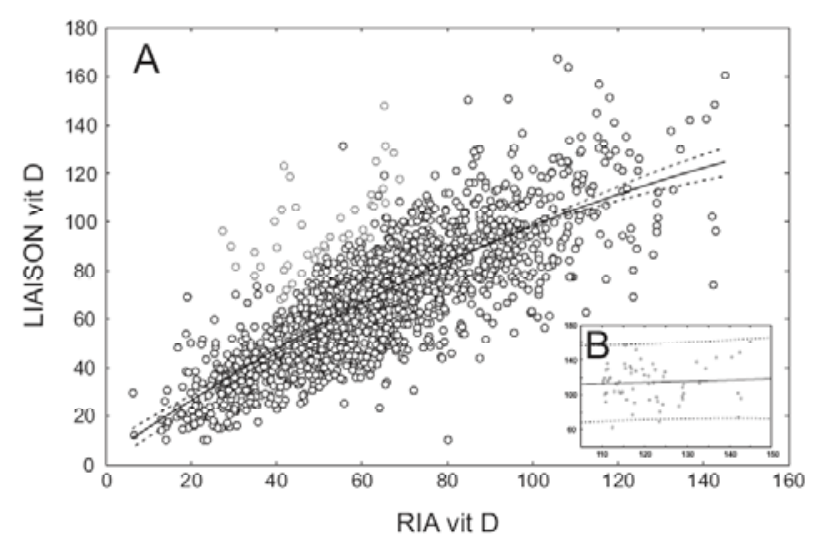

Fig. (2). A. Non-linear quadratic regression of RIA versus LIAISON. Solid line shows line of best fit (see Table $\mathbf{1}$ for parameters). Dashed lines show 95\% confidence limits. B. Linear regression analysis of values greater or equal to $110 \mathrm{nmol} / \mathrm{L}$. Solid line represents regression fit. Dashed lines show $95 \%$ confidence limits.

\section{DISCUSSION}

A common practice when performing a clinical cross over or a method comparison test is to perform multiple tests on a smaller set of between 36-400 samples over a short period of time [19, 20, 27-30]. While this can be sufficient in some circumstances, it can also introduce other possible sources of error. The present study demonstrates that there is value in using a higher number of samples than is commonly used for performing the comparison. In addition, it shows the advantage of conducting the crossover using samples spread over a longer period of time and utilizing several different assay kits of different lots. This would be especially true when comparing across sampling cycles for a long-term survey.

Although the RIA method is regarded as a wellestablished reference method for comparison purposes [19], results from the DEQAS program show that the LIAISON method forms a significant portion of the total submitted values [31]. It is, therefore, important to define the relationship between these two methods. The present study, analysed by weighted Deming regression, showed a good correlation between methods (Spearman $r=0.77$ ) with a slope of $1.14 \pm$ 0.02 (Table 1). These values are slightly higher than a recent comparison analysed by Deming regression using 390 samples, which reported an excellent correlation (Spearman $\mathrm{r}=$ 0.91 ) and a slope of 0.99 [19]. Unlike the data of Wagner et al. [19], the correlation of the present study was improved when a quadratic fit was applied to the data, presumably because of non-linearity at both ends of the data range. The reasons for this non-linearity cannot be determined from the present analysis but the lack of correlation between the two methods at high $25(\mathrm{OH}) \mathrm{D}$ values in our hands is of concern. Part of the difficulty in this part of the curve may lie in the lower signal to noise ratio of response, which would magnify small pipetting errors as well as other relatively minor differences in procedure. From a theoretical perspective (operator reproducibility), the automated system should be more reproducible at this range than the manual RIA kit. In accordance with this principle, we have noticed a decrease in the variance associated with our measurements after switching to the Liaison (RIA difference from DEQAS ALTM -28.5\% to $15.2 \%$; Liaison difference from DEQAS ALTM $-6.3 \%$ to $4.0 \%)$. The significant $\mathrm{Y}$-intercept obtained from regression analysis suggests that at lower $25(\mathrm{OH}) \mathrm{D}$ plasma concentrations, the two methods differ significantly but are more closely related at about $80 \mathrm{nmol} / \mathrm{L}$. The reasons for this are not clear. The antibody used is identical for both methods, but the RIA is an indirect method utilizing a solvent extraction procedure prior to the immunoassay, while the quicker direct LIAISON method skips this step. Whether this accounts for the non-linear response at the higher concentration range is unknown. The non-linearity in response for both methods at the extremes of the response curve appears to influence the relationship between the methods. At the low end of the curve, this resulted in a non-zero intercept, however the direction and magnitude of this deviation cannot be determined from this study.

Bland-Altman analysis showed a significant bias with the LIAISON method giving higher values - a situation opposite to that suggested from the DEQAS program results [32] and one that was higher than that of Wagner et al. [19] illustrating the value of laboratory-specific cross over studies. Some of these differences may be related to differences in RIA and LIAISON response to D2 and D3 in plasma samples. The DEQAS samples may come from a single individual and this 
may include a significant D2 component that can be sample specific. In contrast, the samples from the present survey were obtained from individuals with varied diets, but who are all potentially exposed to supplemental D3 in milk and margarine.

\section{ACKNOWLEDGEMENTS}

This paper is dedicated to the memory of our friend and colleague Dr. Nick Hidiroglou who passed away suddenly on July 17, 2010 at the age of 48. Dr. Hidiroglou was the Health Canada research scientist responsible for fat soluble vitamins. His life's work was dedicated to science and discovery and his contribution to the field of vitamin D research will be greatly missed. His work contributed significantly to the basis of the present investigation. The authors gratefully acknowledge the statistical expertise of Dr. Dietmar Stöckl.

\section{REFERENCES}

[1] Reichel, H.; Norman, A.W. Systemic effects of vitamin D. Annu. Rev. Med., 1989, 40, 71-78.

[2] Lin, J.; Manson, J.E.; Lee, I.M.; Cook, N.R.; Buring, J.E.; Zhang, S.M. Intakes of calcium and vitamin $D$ and breast cancer risk in women. Arch. Intern. Med., 2007, 167(10), 1050-1059.

[3] Gorham, E.D.; Garland, C.F.; Garland, F.C.; Grant, W.B.; Mohr, S.B.; Lipkin, M.; Newmark, H.L.; Giovannucci, E.; Wei, M.; Holick, M.F. Optimal vitamin D status for colorectal cancer prevention: a quantitative meta analysis. Am. J. Prev. Med., 2007, 32(3), 210-216.

[4] Giovannucci, E.; Liu, Y.; Hollis, B.W.; Rimm, E.B. 25hydroxyvitamin $\mathrm{D}$ and risk of myocardial infarction in men: a prospective study. Arch. Intern. Med., 2008, 168(11), 1174-1180.

[5] Pittas, A. G.; Chung, M.; Trikalinos, T.; Mitri, J.; Brendel, M.; Patel, K.; Lichtenstein, A. H.; Lau, J.; Balk, E. M. Systematic review: vitamin D and cardiometabolic outcomes. Ann. Intern. Med., 2010, 152(5), 307-314.

[6] Grant, W.B. Epidemiology of disease risks in relation to vitamin D insufficiency. Prog. Biophys. Mol. Biol., 2006, 92(1), 65-79.

[7] White, J.H. Vitamin D signaling, infectious diseases, and regulation of innate immunity. Infect. Immun., 2008, 76(9), 3837-3843

[8] Mau, J.-L.; Chen, P.-R.; Yang, J.-H. Ultraviolet irradiation increased vitamin D2 content in edible mushrooms. J. Agric. Food Chem., 1998, 46(12), 5269-5272.

[9] DeLuca, H.F. Overview of general physiologic features and functions of vitamin D. Am. J. Clin. Nutr., 2004, 80(6 Suppl), 1689S96S.

[10] Looker, A.C.; Pfeiffer, C.M.; Lacher, D.A.; Schleicher, R.L.; Picciano, M.F.; Yetley, E.A. Serum 25-hydroxyvitamin D status of the US population: 1988-1994 compared with 2000-2004. Am. J. Clin. Nutr., 2008, 88(6), 1519-1527.

[11] Atherton, K.; Berry, D.J.; Parsons, T.; Macfarlane, G.J.; Power, C.; Hypponen, E. Vitamin D and chronic widespread pain in a white middle-aged British population: evidence from a cross-sectional population survey. Ann. Rheum. Dis., 2009, 68(6), 817-822.

[12] Cheng, S.; Massaro, J. M.; Fox, C. S.; Larson, M. G.; Keyes, M. J.; McCabe, E. L.; Robins, S. J.; O'Donnell, C.J.; Hoffmann, U.; Jacques, P.F.; Booth, S.L.; Vasan, R.S.; Wolf, M.; Wang, T.J. Adiposity, cardiometabolic risk, and vitamin D status: the Framingham Heart Study. Diabetes, 2010, 59(1), 242-248.

[13] Jenab, M.; Salvini, S.; van Gils, C. H.; Brustad, M.; ShakyaShrestha, S.; Buijsse, B.; Verhagen, H.; Touvier, M.; Biessy, C.; Wallstrom, P.; Bouckaert, K.; Lund, E.; Waaseth, M.; Roswall, N.; Joensen, A.M.; Linseisen, J.; Boeing, H.; Vasilopoulou, E.; Dilis, V.; Sieri, S.; Sacerdote, C.; Ferrari, P.; Manjer, J.; Nilsson, S.; Welch, A.A.; Travis, R.; Boutron-Ruault, M.C.; Niravong, M.; Bueno-de-Mesquita, H.B.; van der Schouw, Y.T.; Tormo, M.J.; Barricarte, A.; Riboli, E.; Bingham, S.; Slimani, N. Dietary intakes of retinol, beta-carotene, vitamin D and vitamin E in the European Prospective Investigation into Cancer and Nutrition cohort. Eur .J. Clin. Nutr., 2009, 63(Suppl. 4), S150-S178.

[14] McCullough, M.L.; Stevens, V.L.; Patel, R.; Jacobs, E.J.; Bain E.B.; Horst, R.L.; Gapstur, S.M.; Thun, M.J.; Calle, E.E. Serum 25hydroxyvitamin $\mathrm{D}$ concentrations and postmenopausal breast cancer risk: a nested case control study in the Cancer Prevention Study-II Nutrition Cohort. Breast Cancer Res., 2009, 11(4), R64.

[15] Park, S.Y.; Cooney, R.V.; Wilkens, L.R.; Murphy, S.P.; Henderson, B.E.; Kolonel, L.N. Plasma 25-hydroxyvitamin D and prostate cancer risk: the multiethnic cohort. Eur. J. Cancer, 2010, 46(5), 932-936.

[16] Hollis, B.W. Measuring 25-hydroxyvitamin D in a clinical environment: challenges and needs. Am. J. Clin. Nutr., 2008, 88(2), 507S-510S.

[17] Looker, A.C.; Lacher, D.A.; Pfeiffer, C.M.; Schleicher, R.L.; Picciano, M.F.; Yetley, E.A. Data advisory with regard to NHANES serum 25-hydroxyvitamin D data. Am. J. Clin. Nutr., 2009, 90(3), 695.

[18] Institute of Medicine, Dietary Reference Intakes for Calcium, Phosphorus, Magnesium, Vitamin D, and Fluoride. National Academy Press: Washington, DC, 1997.

[19] Wagner, D.; Hanwell, H.E.; Vieth, R. An evaluation of automated methods for measurement of serum 25-hydroxyvitamin D. Clin. Biochem., 2009, 42(15), 1549-1556.

[20] Kimball, S.M.; Vieth, R. A comparison of automated methods for the quantitation of serum 25-hydroxyvitamin $\mathrm{D}$ and 1,25dihydroxyvitamin D. Clin. Biochem., 2007, 40(16-17), 1305-1310.

[21] Binkley, N.; Krueger, D.; Cowgill, C. S.; Plum, L.; Lake, E.; Hansen, K.E.; DeLuca, H.F.; Drezner, M.K. Assay variation confounds the diagnosis of hypovitaminosis D: a call for standardization. $J$. Clin. Endocrinol. Metab., 2004, 89(7), 3152-3157.

[22] Day, B.; Langlois, R.; Tremblay, M.; Knoppers, B.-M. Canadian health measures survey: ethical, legal and social issues. Health Rep., 2007, 18, S37-S51.

[23] Giroux, S. Canadian health measures survey: sampling strategy overview. Health Rep. (Statistics Canada, Catalogue 82-003), 2007, 18(Suppl.), 31-36.

[24] Stockl, D.; Dewitte, K.; Thienpont, L.M. Validity of linear regression in method comparison studies: is it limited by the statistical model or the quality of the analytical input data? Clin. Chem., 1998, 44(11), 2340-2346.

[25] Deming, W. Statistical adjustment of data. John Wiley and Sons: New York, 1943.

[26] Martin Bland, J.; Altman, D. Statistical methods for assessing agreement between two methods of clinical measurement. Lancet, 1986, 327(8476), 307-310.

[27] Roth, H.J.; Schmidt-Gayk, H.; Weber, H.; Niederau, C. Accuracy and clinical implications of seven 25-hydroxyvitamin D methods compared with liquid chromatography-tandem mass spectrometry as a reference. Ann. Clin. Biochem., 2008, 45(Pt 2), 153-159.

[28] Glendenning, P.; Noble, J.M.; Taranto, M.; Musk, A.A. McGuiness, M.; Goldswain, P.R.; Fraser, W.D.; Vasikaran, S.D. Issues of methodology, standardization and metabolite recognition for 25-hydroxyvitamin D when comparing the DiaSorin radioimmunoassay and the Nichols Advantage automated chemiluminescence protein-binding assay in hip fracture cases. Ann. Clin. Biochem., 2003, 40(Pt 5), 546-551.

[29] Glendenning, P.; Taranto, M.; Noble, J.M.; Musk, A.A.; Hammond, C.; Goldswain, P.R.; Fraser, W.D.; Vasikaran, S.D Current assays overestimate 25-hydroxyvitamin D3 and underestimate 25-hydroxyvitamin D2 compared with HPLC: need for assayspecific decision limits and metabolite-specific assays. Ann. Clin. Biochem., 2006, 43(Pt 1), 23-30.

[30] Hollis, B.W. Comparison of commercially available (125)I-based RIA methods for the determination of circulating 25hydroxyvitamin D. Clin. Chem., 2000, 46(10), 1657-1661.

[31] Vitamin D External Quality Assessment Scheme (DEQAS) 25OHD report, Charing Cross Hospital: London, UK, October, 2010.

[32] Carter, G.D.; Berry, J.L.; Gunter, E.; Jones, G.; Jones, J.C.; Makin, H.L.; Sufi, S.; Wheeler, M.J. Proficiency testing of 25hydroxyvitamin D (25-OHD) assays. J. Steroid Biochem. Mol. Biol., 2010, 121(1-2), 176-179. 